\title{
Alternativas a la producción y mercadeo para la carne de conejo en Tlaxcala, México
}

\author{
Rodrigo Olivares Pineda* \\ Manuel Ángel Gómez Cruz* \\ Rita Schwentesius Rindermann* \\ Benjamín Carrera Chávez ${ }^{* *}$
}

Resumen: ${ }^{* * *}$ La cunicultura es una actividad importante en Tlaxcala, en comparación con otras entidades productoras, por lo que se elaboró un diagnóstico técnico-económico sobre ella. Además, se analizaron algunos de los canales de comercialización, para proponer estrategias que faciliten la venta de la carne. Se realizó un estudio técnico de las granjas, complementado con entrevistas semiestructuradas a criadores seleccionados aleatoriamente, a informantes clave de los eslabones de producción y comercialización y visitas periódicas a puntos de venta. Uno de los aspectos típicos de la cunicultura en Tlaxcala es el predominio de un sistema extensivo, combinado con características de otros como el semiintensivo y empresarial. Algunas alternativas para mejorar la comercialización de la carne de conejo son: incrementar la eficiencia en su acopio, generar centros de distribución y puntos de venta, emplear economías de escala y diferenciar el producto que se expende al consumidor.

Palabras clave: cunicultura, Tlaxcala, carne de conejo, sistemas de producción, estrategias de comercialización.

\footnotetext{
* Programa Integración Agricultura Industria (PIAI), Centro de Investigaciones Económicas, Sociales y Tecnológicas de la Agroindustria y la Agricultura Mundial (CIESTAAM), Universidad Autónoma Chapingo (UACH). Km. 38.5 carretera México-Texcoco, C.P. 56230. Chapingo, Estado de México. Correos electrónicos: rolivares77@hotmail.com / rolivaresp77@yahoo.com.mx / ciestaam@avantel.net / rsr@avantel. net

** Administración de Riesgos en la Dirección General de Estudios Agropecuarios y Forestales de la Secretaría de Agricultura, Ganadería, Desarrollo Rural, Pesca y Alimentación (SAGARPA). Correo electrónico:spomos2000@yahoo.com.mx

*** Los autores agradecen los comentarios del doctor Fernando Cervantes Escoto.
} 
Abstract: Given the importance of rabbit farming in Tlaxcala, particularly when compared to other Mexican rabbit producing states, this article describes a technical diagnostic of the activity. The purpose of this diagnostic was to analyze some of the available channels for marketing and sale, and to propose strategies to facilitate sales. In order to conduct this work, technical analyses were conducted on participating farms. These analyses were complemented by semi-structured interviews with randomly selected producers and key informants involved in the chain of production, as well as by periodic visits to locations where rabbit meat is sold. One result of this study is that the rabbit producers in Tlaxcala use a predominantly extensive system of production, while incorporating some elements of semi-intensive or corporate systems. Some alternatives that could improve the marketing and sale of rabbit meat include: increasing the efficiency of product storage; creating distribution centers and points of sale; taking advantage of economies of scale; and differentiating the product that is sold to the consumer.

Key words: rabbit farming, production, rabbit meat, production systems, commercialization strategies, Tlaxcala.

\section{Introducción}

Más de 90 por ciento de la carne que se consume en el mundo es de cerdo, res y aves, sólo 0.5 corresponde a la de conejo. Mientras que en Italia, la demanda per cápita es de 5.3 kilos al año, en México sólo llega a alrededor de 40 gramos. México ocupa el décimo cuarto lugar mundial como productor, con 4200 toneladas, muy inferior a China (500 mil) e Italia (225 mil) (Organización de las Naciones Unidas para la Agricultura y Alimentación, FAO, por sus siglas en inglés, 2007).

Pese a que la cría y consumo del conejo son bajos, la cunicultura es una actividad favorable para campesinos pequeños y medianos en todo el mundo, debido a su fácil manejo, la rapidez para recuperar la inversión y a la posibilidad de generar ingresos modestos durante todo el año. De acuerdo con la FAO, los conejos tienen propiedades únicas como alimento y para el comercio, por ejemplo: 
- La carne es muy nutritiva, con poca grasa y colesterol y es abundante en proteínas ${ }^{1}$

- Como los animales son herbívoros, no compiten con las personas por los alimentos, y se adaptan con facilidad a distintos medios

- Los costos de inversión y mano de obra son pocos, y los miembros más vulnerables de las familias pueden ocuparse de cuidarlos

- Son muy productivos, porque tienen hasta 40 crías al año, en comparación con 0.8 del ganado vacuno y 1.4 del ovino (FAO 1999).

Pese a estas ventajas, el progreso de la cunicultura en México ha sido limitado sobre todo por falta de apoyo oficial, carencia de políticas sanitarias que eviten las epizootias, poco interés en las instituciones de enseñanza e investigación, ausencia de animales mejorados genéticamente, poca difusión del consumo de esta carne y organización precaria entre los productores (García et al. 1998). ${ }^{2}$ Sin embargo, esto no es una situación general, ya que en algunos estados se han canalizado subsidios para el fomento a la producción, organización y creación de estructuras de comercialización, lo que ha mitigado dicha problemática.

En México, el gobierno federal impulsó la cunicultura desde principios de la década de 1970, por medio de un programa de fomento gubernamental (Lebas et al. 1996). La producción aumentó hasta 1981, sin embargo, a partir del año siguiente la actividad decreció, entre otras causas debido a la baja tecnología, mala calidad de los alimentos comerciales y carencia de canales de comercialización (Haro 1987). A finales de 1988 se detectó la presencia de la enfermedad hemorrágica viral (VHD, por sus siglas en inglés) en algunas granjas, por lo que las autoridades zoosanitarias realizaron una campaña con base en cuarentena, inspección, sacrificio, desinfección y sobrevigilancia; este problema provocó, una vez más, un estancamiento en la producción y en la comercialización de la carne.

A partir de 1995, el país se declaró libre de la VHD, por lo que la actividad resurgió en explotaciones comerciales pequeñas, que se dieron a la tarea de explorar y abrir vías nuevas de mercadeo (Mendoza 2001). Para el año 2000, la cunicultura se había extendido a Puebla, Tlaxcala, Michoacán, Hidalgo, Estado de México y Distrito Federal, aunque también existían granjas en Jalisco, Aguascalientes y Tamaulipas.

\footnotetext{
1 Contiene entre 19 y 25 por ciento de proteína, 3 y 6 de grasa y 40 miligramos de sodio por cada 100 gramos (Camps et al. 2001).

2 Todo ello explica también la ausencia de bibliografía científica sobre esta cadena agroalimentaria.
} 
En el caso de Tlaxcala, a partir de 1998 los gobiernos estatal y federal apoyaron la cunicultura con el objetivo de incentivar la ingesta de esta carne entre las familias rurales, para fomentar el autoconsumo y mejorar la calidad de la dieta de sus habitantes; sin embargo, los beneficiados no la comen debido a sus tradiciones, y por ello optaron por venderla en la localidad y la región. En los años siguientes, las autoridades agropecuarias continuaron apoyando la creación de granjas, las cuales dirigieron la carne a los mismos mercados. Para 2004, el incremento constante de la oferta provocó una crisis que causó un descenso significativo de los precios pagados al cunicultor, situación que no sólo perjudicó a los tlaxcaltecas, sino a todo el subsector en los estados del centro del país y se reflejó en sus mercados principales: la Ciudad de México y su área conurbana.

La cunicultura ha estado presente por décadas en la economía alimentaria regional y nacional, y ha sido objeto de políticas públicas aunque de manera relegada. ${ }^{3}$ No obstante, salvo excepciones, en algunas regiones del centro del país no se ha consolidado como una cadena agroalimentaria exitosa.

Por lo ya expuesto, se propusieron los objetivos siguientes para la elaboración del presente estudio: realizar un diagnóstico técnico de la cunicultura en Tlaxcala, analizar algunos de los medios de comercialización en los que incursionaron los criadores y proponer estrategias que mejoren la producción y faciliten la comercialización. La importancia del trabajo radica en el hecho de que presenta un análisis de un sector ganadero prácticamente ausente en la literatura científica. En México predominan ensayos sobre la formulación de proyectos, que no llegan a publicarse y quedan en el ámbito administrativo. Por esta razón se realizó dicho diagnóstico, que puede servir de base para formular políticas públicas y estrategias individuales de los productores, más exitosas de lo que se ha experimentado y conocido en el pasado.

\section{Materiales y métodos}

En el estudio se utilizó el enfoque de cadenas agroalimentarias, propuesto por el Instituto Interamericano de Cooperación para la Agricultura (IICA) en 1996 (Herrera y Burgeois 1996), y actualizado por el International Food Policy Research Institute (IFPRI), en 2006 (Sanders et al. 2006). La cadena se

\footnotetext{
3 Esto consta en el hecho de que la SAGARPA no ofrece información estadística sobre el sector, lo que dificulta su análisis y la formulación de propuestas específicas.
} 
entiende como un conjunto de actores y actividades alrededor de un producto en un espacio dado (Herrera y Burgeois 1996). El enfoque de cadena se refiere al complejo de operaciones de producción, procesamiento, almacenamiento, distribución y comercialización de insumos y bienes agropecuarios y agroforestales, incluidos los servicios de apoyo. ${ }^{4}$ En este caso, la investigación se concentra en la fase de producción y comercialización de conejos en un estado definido, y se analizan los aspectos técnicos, económicos, organizativos y comerciales. Esta dimensión implica considerar diversos procesos productivos, así como las relaciones económicas y comerciales hasta llegar a la demanda final. El objetivo de la metodología utilizada es encontrar y definir elementos de acciones y políticas, para planear ventajas competitivas para el sector. El enfoque de cadenas permite tener una visión amplia de los problemas y un manejo completo de la información, que facilita la identificación de puntos críticos y cuellos de botella, que impiden el avance de la cadena agroalimenticia. Asimismo, apoya la búsqueda de alianzas entre los actores y grupos de interés, para lograr sinergias e innovaciones específicas (Sanders et al. 2006, 3-4).

Se eligió a Tlaxcala porque ha recibido apoyo permanente de las autoridades estatales y federales en el sector cunícola, y por no existir esfuerzos similares recientes en otros estados.

El análisis técnico de las granjas se realizó con base en los diagnósticos elaborados por 10 de los 14 especialistas del Programa de Desarrollo de Proyectos Agropecuarios Integrales (DPAI), que forma parte de otros como el de Fomento Ganadero de la Alianza Contigo, que proporcionó asistencia técnica y capacitación a los cunicultores. El personal del DPAI analizó algunos aspectos de las unidades de producción antes y después de brindar sus servicios. De esta forma, se obtuvieron 41 diagnósticos, que representan 63 por ciento de los grupos existentes. La información se complementó con 18 entrevistas semiestructuradas a criadores seleccionados aleatoriamente y visitas a sus granjas respectivas, donde se utilizó la observación participante (Santoyo et al. 2000, 55-58). Además, se aplicaron entrevistas semiestructuradas a 18 informantes clave de los eslabones de producción y comercialización: funcionarios de la delegación SAGARPA en la entidad, Secretaría de Fomento Agropecuario, representantes y gerente de comercialización de la empresa Toxtitlax, personal técnico, introductores y comercializadores.

Las entrevistas se realizaron entre mayo y septiembre de 2004. Un recorrido adicional en mayo del año siguiente permitió aclarar dudas pendientes.

\footnotetext{
${ }^{4}$ No se incluyó el uso y la comercialización de pieles, por ser insignificante.
} 
El estudio de los canales de comercialización se llevó a cabo mediante visitas periódicas a 20 puntos de venta de carne de conejo (véase cuadro 1), para obtener información sobre precios de compra, venta y volúmenes comercializados. El trabajo se realizó entre agosto y octubre de 2004.

\section{Cuadro 1}

Localización de los puntos de comercialización de la carne de conejo, 2004

\begin{tabular}{|c|c|l|}
\hline $\begin{array}{c}\text { Zonas de la Ciudad } \\
\text { de México }\end{array}$ & $\begin{array}{c}\text { Número de puntos de } \\
\text { venta entrevistados }\end{array}$ & Lugar \\
\hline Centro & 4 & $\begin{array}{l}\text { Mercados públicos: San Juan } \\
\text { y San Cosme }\end{array}$ \\
\hline Oriente, zona conurbana & 6 & $\begin{array}{l}\text { Restaurantes en la carretera México- } \\
\text { Texcoco }\end{array}$ \\
\hline Poniente & 2 & $\begin{array}{l}\text { Restaurantes en la carretera } \\
\text { federal México-Toluca }\end{array}$ \\
\hline Norte, zona conurbana & 6 & $\begin{array}{l}\text { Asadores de carne de conejo } \\
\text { en la vía López Portillo }\end{array}$ \\
\hline Oriente & 2 & $\begin{array}{l}\text { Restaurantes en la autopista México- } \\
\text { Puebla }\end{array}$ \\
\hline Total & 20 & \\
\hline
\end{tabular}

Fuente: elaboración propia, con base en trabajo de campo.

\section{Resultados y discusión}

\section{Situación de la cunicultura en Tlaxcala}

En el estado se encontraron 722 cunicultores organizados en 86 grupos de trabajo, quienes en 2003 recibieron asistencia y capacitación, a través de 14 técnicos adscritos al DPAI. Dichos criadores aglomeraron unos 6 mil semovientes y destinaron al mercado 20 mil, lo cual representa 40 por ciento del inventario y de la producción estatal. ${ }^{5}$

En los años 2000 y 2001, el Programa de Mujeres en el Desarrollo Rural proporcionó los paquetes de conejos; y en los siguientes (de 2002 a 2005), los apoyos para equipo y semovientes se otorgaron a través del Programa de Apoyo a Proyectos de Inversión Rural (PAPIR), de la Alianza para el Campo.

\footnotetext{
${ }^{5}$ El inventario estatal se estima en 15 mil semovientes con 50 mil canales anuales, que en peso representaron alrededor de 65 toneladas de carne (SAGARPA 2004).
} 
Así, conforme maduraron los semovientes, aumentó el número de animales destinados al mercado. Primero, la oferta de conejos fue acaparada por intermediarios, quienes pagaron en promedio 11 pesos por kilo en pie, durante 2001 y 2002 (información obtenida del trabajo de campo).

Ante la carencia de estructuras de comercialización, 19 grupos de trabajo se organizaron para compactar la oferta de los animales. En un principio tuvieron el apoyo de la Fundación Produce Tlaxcala para acopiar, sacrificar y vender los conejos; después, conformaron una integradora para realizar dichas actividades. Ésta fue la antecesora de Toxtitlax, empresa creada para comercializar exclusivamente los conejos de sus socias. Sin embargo, cuando la demanda lo requiere, compra animales de cunicultores independientes. El precio que la compañía pagó en 2003 y 2004 por conejo vivo fue de 17 pesos por kilo; en 2005 subió a 18 (Ibid.).

Para la consolidación de Toxtlitlax y de su estructura organizativa, se contó con subsidios del Programa Fortalecimiento de Empresas y Organización Rural (PROFEMOR) de la Alianza para el Campo, de tal forma que en 2003 recibió 250 mil pesos, que fueron destinados a pagar sus costos de gerencia y administración (SAGARPA 2004).

A pesar de las expectativas generadas, entre mayo y septiembre de 2004, el exceso de conejos en el estado y la disminución de la demanda de carne causaron la saturación de los canales de mercadeo, por lo que la empresa ya no los acopió y las granjas no pudieron desplazar su producto. Esta situación provocó que el precio pagado por los intermediarios bajara a 12 pesos por kilo en promedio, lo que ocasionó el cierre de algunas granjas.

Aspectos técnicos

En México existen tres sistemas de producción cunícola bien definidos y caracterizados (véase cuadro 2). En el caso de Tlaxcala, predomina el uso del extensivo, mezclado con algunos componentes del semiintensivo e intensivo.

Aunque la cunicultura se practica en todo el estado, en los diagnósticos realizados se observó una mayor concentración de granjas en los municipios de Altzayanca, Ixtacuixtla, Cuapiaxtla, Huamantla y Españita. Los grupos de trabajo están compuestos por 8.2 integrantes en promedio, cada uno tiene 12.7 semovientes, con una relación de 11 hembras por semental.

\section{Manejo reproductivo}

Todas las granjas utilizan monta natural, en la que la hembra se presenta al macho. Sin embargo, sólo 76.2 por ciento de los productores revisan la colo- 


\section{Cuadro 2}

Características de los sistemas cunícolas de producción en México

\begin{tabular}{|c|c|c|c|}
\hline \multirow[b]{2}{*}{ Aspectos } & \multicolumn{3}{|c|}{ Sistema de producción } \\
\hline & Traspatio o extensivo & Semiintensivo & Intensivo o empresarial \\
\hline Tipo de cubrición & Monta natural & Monta natural & $\begin{array}{l}\text { Monta natural / } \\
\text { inseminación artificial }\end{array}$ \\
\hline Monta & Posdestete & 15 días posparto & 3-9 días posparto \\
\hline Destete & $\begin{array}{l}\text { A los } 35 \\
\text { días posparto }\end{array}$ & $\begin{array}{l}\text { A los } 35 \text { días } \\
\text { posparto }\end{array}$ & A los 30 días posparto \\
\hline Instalaciones & Ambiente natural & Ambiente natural & Ambiente controlado \\
\hline Alimentación & $\begin{array}{l}\text { Mixta: alimento } \\
\text { balanceado, } \\
\text { desperdicios de cocina, } \\
\text { subproductos agrícolas }\end{array}$ & $\begin{array}{l}\text { Alimento comercial } \\
\text { único }\end{array}$ & $\begin{array}{l}\text { Alimento comercial } \\
\text { diferenciado por } \\
\text { etapa productiva }\end{array}$ \\
\hline Manejo sanitario & $\begin{array}{l}\text { No se realizan } \\
\text { desparasitaciones }\end{array}$ & $\begin{array}{l}\text { Desparasitaciones } \\
\text { internas y externas }\end{array}$ & $\begin{array}{l}\text { Desparasitaciones } \\
\text { internas y externas }\end{array}$ \\
\hline Identificaciones & $\begin{array}{l}\text { Animales sin } \\
\text { identificación }\end{array}$ & $\begin{array}{l}\text { Animales tatuados } \\
\text { y con tarjeta de } \\
\text { control }\end{array}$ & $\begin{array}{l}\text { Animales tatuados y } \\
\text { con tarjeta de control }\end{array}$ \\
\hline Uso de registros & Sin uso de registros & $\begin{array}{l}\text { Registros } \\
\text { productivos }\end{array}$ & Registros productivos \\
\hline $\begin{array}{l}\text { Destino de la } \\
\text { producción }\end{array}$ & $\begin{array}{l}\text { Principalmente } \\
\text { autoconsumo }\end{array}$ & $\begin{array}{l}\text { Venta de animal } \\
\text { vivo y en canal }\end{array}$ & $\begin{array}{l}\text { Venta de animal vivo y } \\
\text { en canal }\end{array}$ \\
\hline Producción & $\begin{array}{l}30 \text { gazapos/hembra } \\
\text { /año }\end{array}$ & $\begin{array}{l}45 \text { gazapos/hembra } \\
\text { / año }\end{array}$ & $\begin{array}{l}55 \text { gazapos/hembra } \\
\text { /año }\end{array}$ \\
\hline $\begin{array}{l}\text { Mortalidad en } \\
\text { engorda }\end{array}$ & $>10 \%$ & $<10 \%->5 \%$ & $<5 \%$ \\
\hline
\end{tabular}

Fuente: elaboración propia, con base en Mendoza (2001).

ración de la vulva como método para la detección de estros; después de la cubrición de la hembra, 59.5 por ciento de los cunicultores realizan diagnóstico de gestación, para corroborar si ésta quedó gestante. En promedio, el nidal se coloca a la hembra a los 28 días de gestación. Este manejo es posible, ya que en 73.8 por ciento de las granjas se llevan registros productivos, en los que se anota la fecha de monta y se calendarizan otras actividades. Después del parto, la hembra se presenta al macho a los 15 días; el destete se realiza a los 33 días de edad de los gazapos. En general, las jaulas están numeradas, lo que facilita manipular a los animales. 
Manejo alimenticio

La base de la comida para los conejos es alimento balanceado comercial, pero no hay una diferenciación de éste por etapa productiva. Además, 35.7 por ciento de los criadores emplean subproductos agrícolas para complementar la dieta de los animales en engorda y abaratar costos. Esto provoca que las canales no sean homogéneas, pues con esta forma de alimentación se incrementa el tiempo de engorda, en detrimento de la calidad de la carne.

Manejo genético

Los fenotipos utilizados en las granjas son Nueva Zelanda, Chinchilla, California y Azteca Negro, con una tendencia de producir animales híbridos para carne. Sin embargo, no se ha realizado el manejo adecuado para la selección de vientres de reemplazo, aunque se llevan fichas de registro. La información no es procesada para seleccionar a los animales por sus parámetros o para eliminar vientres poco aptos para la reproducción. Esto se refleja en que 91.5 por ciento de las granjas compra sus semovientes en otras explotaciones, 9.5 selecciona los vientres y 85.7 no tiene criterios definidos para eliminar semovientes.

Infraestructura

Los conejares están hechos de materiales reciclados (costales, lámina, madera), lo que no impide que se proporcionen las condiciones necesarias de confort. Las jaulas disponibles son del tipo europeo y los comederos y bebederos son recipientes acondicionados para este fin, pocas granjas utilizan los automáticos.

Manejo sanitario

Los problemas más frecuentes son neumonías, sarna y enteritis. Para ello existen medidas preventivas: 76.2 por ciento de los productores desparasita en forma externa a los animales, mientras que 66.6 lo hace internamente; asimismo, a través del manejo del ambiente en el conejar, se busca incidir sobre dificultades respiratorias.

Aspectos económicos

El costo de producción del kilogramo de conejo vivo en el sistema de traspatio fue de 18.40 pesos en 2004, si se toman en cuenta los gastos en ali- 
mento, medicamentos, reemplazos y mano de obra, mientras que en el semiintensivo fue de 16.40 y en el intensivo de 15.50 pesos. Por lo general, los costos calculados por los cunicultores son más bajos, pues no consideran el de la mano de obra familiar, además no llevan un registro de los egresos e ingresos de las granjas (véase cuadro 3).

\section{Cuadro 3}

Utilidad bruta de una granja cunícola con diez vientres en diferentes sistemas de producción en Tlaxcala, 2004

\begin{tabular}{|l|r|r|r|}
\hline Sistema de producción & Traspatio & Semiintensivo & Intensivo \\
\hline Costos de producción anual (\$) & 11600.00 & 14980.00 & 16230.00 \\
Gazapos destetados al año (no) & 350 & 490 & 550 \\
Mortalidad (\%) & 10 & 7 & 5 \\
Gazapos finalizados al año (no) & 315 & 456 & 523 \\
Peso promedio del animal finalizado (kg) & 2.0 & 2.0 & 2.0 \\
Carne producida al año (kg) & 630 & 910 & 1,046 \\
Precio de venta (\$) & 17.00 & 17.00 & 17.00 \\
Ingreso bruto anual (\$) & 10710.00 & 15504.00 & 17782.00 \\
Utilidad anual $(\$)$ & -890.00 & 524.00 & 1552.00 \\
\hline
\end{tabular}

Fuente: elaboración propia, con base en información colectada en campo, 2004.

Asimismo, la utilidad podría incrementarse si vendieran los animales en canal o procesaran y expendieran la carne ya preparada para consumo. En el caso de la venta en canal, ${ }^{6}$ el ingreso bruto se incrementaría a 13230 pesos en traspatio, 19152 y 21966 pesos, respectivamente para los sistemas semiintensivo e intensivo.

Según las condiciones descritas, es difícil que las granjas compitan en el mercado, ya que su manejo es ineficiente y alto su costo de producción. En este esquema es factible producir carne para autoconsumo, pero paradójicamente los cunicultores tlaxcaltecas no la comen.

\section{Aspectos organizativos}

Los criadores están organizados en grupos de trabajo, con el fin de cumplir con el requisito para acceder a los apoyos y a la capacitación otorgados por

\footnotetext{
${ }^{6}$ Con un rendimiento de 60 por ciento en canal y un precio de venta de 35 pesos por kilo.
} 
los programas de la Alianza para el Campo. Es por ello que no existen organizaciones para la producción, y la compra de insumos se hace de manera individual, por lo que no se realizan economías de escala.

\section{Análisis del mercado}

La carne de conejo se consume todo el año, sin embargo, su poca oferta de septiembre a abril favorece que el mercado absorba y desplace la producción existente, aunado a esto la demanda se incrementa en noviembre y diciembre, por cuestiones culturales, ${ }^{7}$ disminuye en enero ${ }^{8}$ y se mantiene de febrero a abril. De mayo a agosto hay mayor oferta, ${ }^{9}$ lo que provoca la saturación del mercado y, por ende, la dificultad para su comercialización. Los precios de la carne también fluctúan en función de la demanda, y de la zona de producción; por ejemplo, el máximo del conejo vivo fue de 19.50 pesos por kilo al poniente de la Ciudad de México en septiembre (Solís 2004), en contraste con los 12 pesos que pagaron los intermediarios en Tlaxcala ese mismo mes, en 2004 (información obtenida del trabajo de campo).

De los productores, 30.10 por ciento vende a intermediarios de la región, quienes visitan las granjas y compran los animales que necesitan. Estos agentes pagan de contado (en promedio 16.20 pesos por kilo en 2004); 69.90 por ciento de las granjas vendió a Toxtitlax, la cual pagó 17, en pie; con el inconveniente de que el pago fue posterior a la venta y en las oficinas de la empresa. Sólo 9.5 por ciento de los criadores ha intentado diversificar su mercado, y llevar la carne al consumidor final. Otra vía de comercialización de conejo vivo son los mercados regionales (López et al. 2000) de San Martín Texmelucan, Puebla, y de Chalco, Estado de México, en los que el volumen semanal ofertado oscila entre 800 y 2 mil animales en el primero y entre 80 y 100 en el segundo; el precio pagado en 2003 fluctuó entre 15.0 y 16.5 pesos por kilo en pie, en dichos lugares (información obtenida del trabajo de campo).

Los intermediarios que compran conejo en Tlaxcala lo comercializan en canal en los puntos de venta de la Ciudad de México y área conurbana. Estos nichos de mercado podrían ser atractivos para los cunicultores tlaxcaltecas,

\footnotetext{
7 El consumo de platillos elaborados con carne de conejo aumenta por la abundancia de eventos sociales en estos dos meses.

8 El decremento se puede atribuir a la "cuesta de enero".

9 Las conejas responden al estímulo luminoso, y entran en celo cuando la cantidad de horas luz es mayor. Esto provoca que haya más partos y mayor producción, aun si el manejo técnico es deficiente.
} 
sin embargo, para acceder a ellos es necesario compactar la oferta y generar un producto de calidad homogénea.

Algunos restaurantes de comida internacional y ciertas cadenas de tiendas de autoservicio incluyen la carne de conejo entre las opciones que ofrecen. No obstante, estos establecimientos exigen requisitos estrictos en términos de tiempo y forma de entrega (homogeneidad, calidad de la canal, volumen, facturación, presentación, producto para promociones), que pocos cunicultores individuales pueden cumplir, por lo que la organización representa una alternativa para incursionar en estos mercados potenciales, y obtener una diferencia sustancial en precios (véase cuadro 4).

\section{Cuadro 4}

Puntos de venta principales de carne de conejo en México, en 2004

\begin{tabular}{|c|c|c|c|c|}
\hline Punto de venta & Presentación & $\begin{array}{c}\text { Animales } \\
\text { comercializados } \\
\text { a la semana }\end{array}$ & $\begin{array}{c}\text { Precio de compra } \\
\text { animales en pie } \\
\text { (pesos por kilo) }\end{array}$ & $\begin{array}{c}\text { Precio de } \\
\text { venta en canal } \\
\text { (pesos por kilo) }\end{array}$ \\
\hline Mercado público & $\begin{array}{l}\text { Animal fresco o } \\
\text { congelado, } \\
\text { eviscerado, con } \\
\text { cabeza y patas; } \\
\text { peso de venta, } \\
\text { de } 1.5 \text { a } 2.0 \text { kilos }\end{array}$ & 1200 & $\begin{array}{l}18.50 \text { a } 20.00 \\
\text { kilos, animales } \\
\text { en pie. } \\
32.00 \text { kilos, } \\
\text { en canal }\end{array}$ & $40-45$ \\
\hline $\begin{array}{c}\text { Tiendas de } \\
\text { autoservicio }\end{array}$ & $\begin{array}{l}\text { Animal congelado, } \\
\text { empaquetado, } \\
\text { eviscerado, con } \\
\text { cabeza y sin patas; } \\
\text { peso promedio } \\
\text { de } 1.3 \text { kilos }\end{array}$ & 5000 a 7000 & $\begin{array}{c}40 \text { a } 44.5 \text { kilos. } \\
\text { Se maneja un } \\
\text { porcentaje de } \\
\text { descuento para } \\
\text { promociones }\end{array}$ & $50-55$ \\
\hline Restaurantes & $\begin{array}{l}\text { Animal congelado } \\
\text { o fresco, eviscerado, } \\
\text { con cabeza y sin } \\
\text { patas; peso de la } \\
\text { canal, variable }\end{array}$ & No determinado & $\begin{array}{c}40 \text { a } 45 \text { kilos, } \\
\text { en canal }\end{array}$ & Variable \\
\hline Asaderos & $\begin{array}{l}\text { Animal fresco, con } \\
\text { cabeza y sin patas; } \\
\text { peso promedio } \\
\text { de } 1.2 \text { kilos }\end{array}$ & 600 & 45 la pieza & $\begin{array}{c}100-130 \text { el } \\
\text { conejo entero }\end{array}$ \\
\hline
\end{tabular}

Fuente: elaboración propia, con base en trabajo de campo e informantes clave, 2004.

Un problema clave en la cunicultura tlaxcalteca es que no se está generando un producto homogéneo y de calidad, que responda a las necesida- 
des del mercado. La canal de conejo es alargada, magra, sin grasa y con poca conformación de las piezas, lo cual puede deberse a la calidad genética de los predecesores o a la alimentación, en contraposición con la canal corta, magra, con grasa, amplitud torácica y la conformación de las piezas que demanda el mercado.

\section{Alternativas para mejorar los sistemas de producción}

Es importante concientizar a los criadores para producir un conejo de más calidad (mayor compactación de la canal, más magro, etcétera) y homogeneidad; para esto es necesario mejorar los aspectos técnicos que presentan deficiencias, y llevar un control de los parámetros productivos y reproductivos de los semovientes, que permita seleccionar animales con las características deseadas.

La alimentación de los conejos en engorda se refleja en la calidad de la canal, por lo que es necesario diferenciar los mercados a los que se va a ofertar la carne realmente demandada. A los compradores que requieran un animal muy magro y de corta edad, se les debe canalizar conejo alimentado con concentrados comerciales; mientras que a los menos exigentes se les puede surtir con animales alimentados con concentrados y subproductos agrícolas. La diferenciación del producto desde la granja permitirá la planeación y comercialización de las canales, ya que se conocerá la cantidad de animales que se obtendrán y sus características, para ofertar a precios y compradores diferenciados.

El papel de los técnicos es fundamental para lograr la consolidación productiva de las granjas, por lo que es necesario generar un programa de estímulos en función de los resultados obtenidos en los conejares que reciben asistencia. Los recursos para los estímulos podrían manejarse como un porcentaje del incremento en productividad.

\section{Alternativas para la comercialización}

Eficiencia en el acopio del producto

La planificación de la producción permitirá programar el acopio del conejo vivo, y establecer rutas semanales o quincenales para llevar los animales al rastro para sacrificarlos y luego distribuirlos. El sacrificio en rastros del tipo inspección federal (TIF) y después en los que cumplan con los requisitos de inocuidad podrá aumentar las ventas en supermercados, hoteles y cadenas de restaurantes. 
Centros de distribución y puntos de venta

La distancia es una limitante para los productores de Tlaxcala, quienes busquen en forma individual competir en el mercado de la Ciudad de México, aunado a esto, son pocos los que compran grandes volúmenes (tiendas de autoservicio, por ejemplo). Por esta situación es necesario crear rutas de distribución y puntos de venta nuevos, de tal forma que la carne de conejo esté más cercana al consumidor. Se podría establecer alianzas estratégicas con otros expendios (carnicerías, pollerías) para que exhiban y vendan esta carne.

Utilización de economías de escala

Si se parte de que la organización de los productores es la adecuada, se puede programar la compra de insumos de todos los de una región, en función de sus necesidades. Además, según el volumen comprado, se puede negociar un mejor precio; los insumos podrían transportarse a un punto estratégico, donde se almacenarían, y después se determinaría una ruta para la entrega, con lo que se abarataría el costo del traslado. Asimismo, a través de la organización, se puede acceder a asistencia técnica, capacitación, apoyos gubernamentales y servicios financieros, entre otros.

Generación de un producto diferenciado

Conejo guisado listo para comer

Las tendencias laborales han orillado a la gente a adquirir comida ya preparada cerca de su centro de trabajo, por lo que su consumo en casa ha disminuido (United States Department of Agriculture, USDA 2002). Por tanto, es necesario ofrecer un alimento de buen sabor y apariencia, que pueda comprarse con facilidad (por ejemplo, en tiendas de autoservicio), y cuya preparación no sea una limitante. En este sentido, la presentación de la carne de conejo cocinada y empacada, que sólo exija el uso del horno de microondas o de la sartén, es una opción viable para no repetir el mismo menú en poco tiempo. La estrategia exige mayor procesamiento agroindustrial de la canal, sin embargo, esto se compensaría por el precio final.

Una alternativa a futuro puede ser la producción orgánica de conejos, ${ }^{10}$ pero aún no existen experiencias en México. Para ello se requiere un sistema cerrado, desde el cultivo del forraje, la selección genética de los anima-

10 Los autores tratan este tema en otro trabajo todavía no publicado. 
les y medidas preventivas de salud, hasta criterios sustentables de comercialización (venta de preferencia local).

\section{Conclusiones y recomendaciones generales}

El estudio de la cunicultura en Tlaxcala permite llegar a algunas conclusiones y hacer las recomendaciones siguientes:

- Establecer una estrategia comercial para promover la carne de conejo entre los consumidores potenciales, asimismo diversificar las presentaciones en que llega al comprador final y los puntos de venta.

- Favorecer la actualización constante de los técnicos y supervisar el trabajo realizado, de tal forma que exista un efecto real en las unidades de producción en aspectos técnicos. Se recomienda que la SAGARPA certifique a los especialistas.

- Canalizar los apoyos a través del DPAI y el PAPIR en forma integral, conjuntando los apoyos físicos (equipo, semovientes) con la asistencia técnica y la capacitación, y dar prioridad a los grupos ya conformados para consolidarlos.

- Generar una cultura de la calidad, que permita diferenciar la carne por la conformación de la canal, su aspecto y precio. En este sentido, la producción orgánica es una opción para diferenciar el producto y, de esta forma acceder a nichos de mercado específicos.

En México existen enfermedades como las vacas locas y la gripe aviar, que ponen en peligro a la salud humana y a la perspectiva económica de los productores de carnes tradicionales. Dichos acontecimientos, que por la apertura comercial y el aumento de los flujos de mercancías se van a repetir y son difíciles de controlar, pueden favorecer en el futuro el incremento de la producción y del hábito de consumo en México de carnes no tradicionales y saludables, como la de conejo. Por ello, se concluye que debería fomentarse todavía más la cunicultura.

Recibido en julio de 2007

Revisado en febrero de 2008 


\section{Bibliografía}

Camps, J. y J. C. de Pedro. 2001. Conejo: la carne sana y dietética. Ciclo internacional de conferencias en cunicultura empresarial. UACH.

FAO. 1999. El conejo: cría, sanidad y producción. Roma: FAO.

FAO-Base de datos estadísticos de la FAO (FAOSTAT). 2007. Base de datos. http: //www.fao.org

García, J. C., A. Pró M., C. M. Becerril P., M. E. Suárez, O. J. I. Cortés F., y M. J. González A. 1998. Diagnóstico de la producción y consumo de la carne de conejo en la población de Xocotlán, Texcoco, Estado de México. Memorias del primer Congreso de cunicultura de las Américas. Montecillo, México.

Haro, A. E. 1987. Seminario Situación y perspectivas de la cunicultura en México. UACH, Departamento de Zootecnia.

Herrera D., y Burgeois R. 1996. Enfoque participativo para el desarrollo de la competitividad de los sistemas agroalimentarios. CADIAC. Cadenas y diálogo para la acción. San José, Costa Rica: Instituto Interamericano de Cooperación para la Agricultura.

Lebas, F., P. Coudert, R. Rouvier y H. Rochambeau. 1996. El conejo: cría y patología. Colección FaO: Producción y Sanidad Animal.

López, M., H. Lozada, R. Soriano, J. Vieyra, J. Cortés y L. Arias. 2000. Storing Centres as a Strategy for the Commercialisation of Rabbit's Meats in the Southeast of Mexico City. $7^{\circ}$ World Rabbit Congress. Valencia.

Mendoza, B. 2001. Situación de la cunicultura en México. Ciclo internacional de conferencias en cunicultura empresarial. UACH.

Olivares, P. R. 2002. Investigación de mercado y análisis de la comercialización de la carne de conejo en la Ciudad de México y zona conurbada. Tesis de maestría en Ciencias, UACH.

Osechas, D., y L. M. Becerra Sánchez. 2006. Producción y mercadeo de carne de conejo en el estado. Trujillo, Venezuela. Revista Científica XvI (2): 129135. 
SAGARPA y Secretaría de Fomento Agropecuario del Estado de Tlaxcala. 2004. Evaluación Alianza Contigo 2003. Informe de evaluación estatal del Programa Fomento Ganadero.

Sanders, Arie, Angélica Ramírez y Lilián Morazán. 2006. Cadenas Agrícolas en Honduras. Desarrollo socioeconómico y ambiente. Escuela Agrícola Panamericana. Zamorano, Honduras. Documento presentado ante la IFPRI.

Santoyo, H., P. Ramírez P. y M. Suvedi. 2000. Manual para la evaluación de programas de desarrollo rural. México: Inca Rural, Michigan State University, UACH, CIESTAAM.

Solís, J. C. 2004. Comercialización del conejo en México. Precongreso del $6^{\circ}$ Congreso Mundial de Cunicultura. Tlaxcala.

USDA. 2002. Agricultural Baseline Projections to 2011. Office of the Chief Economist. U. s. Department of Agriculture. Prepared by the Interagency Agricultural Projections Committee. Staff Report WAOB-2002-1. Washington. 
